\title{
Simulated Annealing Based Multiuser Detection for Synchronous SDMA System
}

\author{
Jinhuan Xia, Tiejun Lv, Member, IEEE, Xiang Yun, Xinghui Su and Shaoshi Yang \\ School of Information and Communication Engineering, \\ Beijing University of Posts and Telecommunications, \\ Beijing, China \\ E-mails: \{xiajinhuan, lvtiejun\}@gmail.com
}

\begin{abstract}
In this treatise, a novel Simulated Annealing (SA) based Multi-User Detection (MUD) is proposed in synchronous Space Division Multiple Access (SDMA) system. SA MUD modifies experiential Cooling Schedule (CS) of traditional SA algorithm according to its use in MUD. Moreover, in order to ensure sufficient diversity acquired in the whole Markov chain and to prevent from being trapped at local optima, Uniform Mutation (UM) based trial vector generation scheme is brought forward. In addition, the optimal solution recording scheme is also invoked in case of being lost during cooling process. Simulation results illustrate that in comparison with Genetic Algorithm (GA) MUD in the same simulation conditions, without turbo processing and soft-information, SA MUD proposed in this paper performs better, approaching the performance of Maximum Likelihood (ML) MUD and imposes lower complexity.
\end{abstract}

\section{INTRODUCTION}

In the family of MUD, Least Squares (LS) and Minimum Mean Square Error (MMSE) are classic linear detection schemes, exhibiting low complexity but limited performance. By contrast, ML MUD can provide the best performance by invoking an exhaustive search, whose computational complexity increases exponentially with the number of simultaneous users supported by the Multiple Input-Multiple Output (MIMO) system, so that its implementation in high-user-loaded scenario is strongly prohibited. As a compromise between performance and complexity, a range of suboptimal MUDs are proposed in many literatures [1], e.g. Successive Interference Cancellation (SIC) and Parallel Interference Cancellation (PIC), which present better performance than LS and MMSE at the cost of sustainable complexity increase. Moreover, most of the above-mentioned techniques were employed in the system, where the number of users is less than or equal to that of receivers, referred to here as the underloaded or fully loaded scenarios, respectively [2]. Whereas, in practical applications, the number of users usually exceeds that of receivers, which is regarded as a rank-deficient scenario, although some MUDs are modified to be fit for such scenario, the performance will be catastrophically degraded with the increase of the number of users.

GA has been employed for solving many optimization problems in numerous fields. GA and Hybrid GA (HGA)

This research has been supported by Program for New Century Excellent Talents in University (NCET) under Grant No. NCET-06-0091. are proposed as suboptimal MUD techniques in Code Division Multiple Access (CDMA) systems in [3] and [4], [5], respectively. Furthermore, GA was introduced into SDMA and Orthogonal Frequency Division Multiplexing (OFDM) systems in [6]-[8]. Unfortunately, GA doesn't perform as well as [6]-[8] in the SDMA system without any auxiliary techniques, e.g. Turbo Trellis Coded Modulation (TTCM), turbo processing and soft-information.

SA has been shown to be another efficient method for exploring NP-hard problems and complex nonlinear search spaces. As it is well known, MUD was rendered as a challenging multidimensional and combinatorial optimization problem. Against this background, an SA based MUD scheme is proposed in this contribution. SA MUD modifies experiential CS of traditional SA algorithm according to its use in MUD. Firstly, optimization metric for SA MUD is formulated from the signals received by multiple antennas of Base Station (BS). Secondly, the initial temperature is determined by the acceptance rate of the worst solution to the best one, both of which are estimated from a population generated randomly from an initial solution assisted by MMSE MUD. Thirdly, twofold stopping criteria are betaken to attain a compromise between Bit Error Rate (BER) performance and calculation complexity. Moreover, in order to ensure sufficient diversity acquired in the whole Markov chain and to prevent from being trapped at local optima, Uniform Mutation (UM) based trial vector generation scheme is brought forward. In this treatise the optimal solution is proposed to be recorded in case of being lost during cooling process. Our simulation results show that SA MUD outperforms GA MUD in synchronous SDMA systems without coding and iteration gains, and is capable of approaching the performance attained by ML MUD.

Notation: Transpose and Hermitian transpose of a vector or matrix are denoted by $(\cdot)^{T}$ and $(\cdot)^{H}$, respectively. $\lceil\cdot\rceil$ represents ceiling operation. Furthermore, vectors are denoted by bold lowercase letters and bold uppercase letters denotes matrices.

\section{SYSTEM MODULE}

\section{A. SDMA MIMO channel module}

We consider an uplink multiuser narrowband SDMA MIMO system, in which the transmitted signals of $L$ simultaneous uplink mobile users are received by the $P$ different receiver 


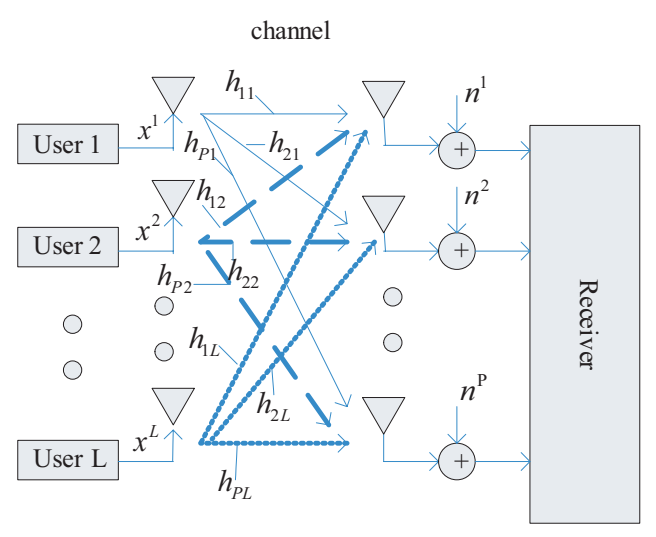

Fig. 1. Schematic of the SDMA uplink MIMO channel model, where each of the $L$ users is equipped with a single transmit antenna and the BS's receiver is assisted by a $P$-element antenna array.

antennas of the BS, and each mobile user is equipped by a single transmit antenna. The system model is depicted in Fig.1. The whole system is assumed in synchronous status, and at the sampling time $k$, the signal received by the $P$-element BS antenna array is constituted by the superposition of the independently faded signals associated with the $L$ mobile users and contaminated by the AWGN noise, expressed as

$$
\mathbf{y}=\mathbf{H x}+\mathbf{n},
$$

where the $(P \times 1)$-dimensional vector $\mathbf{y}$, the $(L \times 1)$-dimensional vector $\mathbf{x}$ and the $(P \times 1)$-dimensional vector $\mathbf{n}$ are the received, transmitted and noise signals, respectively, and $\mathbf{H}$ is a $(P \times$ $L$ )-dimensional channel matrix. Here the sampling time $k$ for each vector is omitted for the sake of notational convenience. Specifically, the vectors $\mathbf{x}, \mathbf{n}, \mathbf{y}$ and matrix $\mathbf{H}$ are given by

$$
\begin{gathered}
\mathbf{x}=\left(x^{1}, x^{2}, \ldots, x^{L}\right)^{T}, \\
\mathbf{n}=\left(n^{1}, n^{2}, \ldots, n^{P}\right)^{T}, \\
\mathbf{y}=\left(y^{1}, y^{2}, \ldots, y^{P}\right)^{T}, \\
\mathbf{H}=\left(\begin{array}{cccc}
h_{11} & h_{12} & \cdots & h_{1 L} \\
h_{21} & h_{22} & \cdots & h_{2 L} \\
\vdots & \vdots & \ddots & \vdots \\
h_{P 1} & h_{P 2} & \cdots & h_{P L}
\end{array}\right) .
\end{gathered}
$$

In (1) to (5), we assume that the AWGN noise signal $n^{p}(p=1, \ldots, P)$ exhibits a zero-mean and a variance of $\sigma_{n}^{2}$, and $h_{i j}(i=1, \ldots, P ; j=1, \ldots, L)$ represents the channel transfer coefficient associated with the propagation link connecting the $i^{\text {th }}$-element BS antenna array and the $j^{\text {th }}$ user's antenna, which is independent, stationary, and complex Gaussian distributed process with zero-mean and unit variance.

\section{B. MMSE MUD and ML MUD}

In the MMSE-based MUD, with the aid of the linear MMSE combiner, the transmitted signals of the different users are estimated. More specifically, the estimated signal vector $\hat{\mathbf{x}} \in \mathbb{C}^{L \times 1}$ is generated from the received signal vector $\mathbf{y}$, as follows [1]

$$
\hat{\mathbf{x}}_{M M S E}=\mathbf{W}_{M M S E}^{H} \mathbf{y},
$$

where $\mathbf{W}_{M M S E} \in \mathbb{C}^{P \times L}$ is the MMSE-based weigh matrix given by [1]

$$
\mathbf{W}_{M M S E}=\left(\mathbf{H H}^{H}+\sigma_{n}^{2} \mathbf{I}\right)^{-1} \mathbf{H},
$$

where $\mathbf{I}$ is the identity matrix.

ML MUD [1] is the optimal scheme, which invokes exhaustive search, and requires $2^{m L}$ evaluations of the Objective Function Metric (OFM) of (8) for finding the most likely transmitted symbol vector $\hat{\mathbf{x}}_{M L}$, and

$$
\hat{\mathbf{x}}_{M L}=\arg \left\{\min _{\breve{\mathbf{x}} \in M^{L}}\|\mathbf{y}-\mathbf{H} \breve{\mathbf{x}}\|_{2}^{2}\right\},
$$

where the set of $M^{L}$ containing all trial-vectors is defined by [1]

$$
M^{L}=\left\{\breve{\mathbf{x}}=\left(\begin{array}{c}
\breve{x}^{1} \\
\vdots \\
\breve{x}
\end{array}\right) \mid \breve{x}^{1}, \ldots, \breve{x} \in M_{C}\right\},
$$

where $M_{C}$ denotes the set covering the $2^{m}$ number of legitimate complex constellation points with the specific modulation scheme deployed, and $m$ denotes the number of bits per symbol.

\section{SIMULATED ANNEALING ALGORITHM BASED MULTIUSER DETECTION}

ML MUD is regards as NP-complete, and the computational complexity increases exponentially with the number of simultaneous users loaded by the system. Fortunately, inherent principle and parallel calculation aptness of SA make it an effective technique solving NPC problems. Fig.2 shows a flowchart of the SA MUD. As it is well known, SA algorithm is an iterative Markov chain Monte Carlo method, and its gradual convergence implies that [10], only if the total length of Markov chain $L_{t o t} \rightarrow \infty$, can the optimal solution be reached. For the sake of practicability, SA should return the suboptimal solution in finite calculations at the expense of little BER performance degradation, which can be realized by contriving appropriate CS. CS as a set of crucial parameters controlling the whole SA algorithm has obtained several experiential values. In this section, UM based trial vector generation scheme is proposed and relevant CS is designed according to the application of SA in MUD. More details are described as follows: 


\section{A. Optimization Metric for SA MUD}

Inspired by (8), the OFM for SA MUD is defined as

$$
f(\mathbf{x})=\sum_{p=1}^{P}\left|y^{p}-\mathbf{h}_{p} \mathbf{x}\right|^{2},
$$

where $y^{p}$ is the received symbol at the input of the $p^{\text {th }}$ element BS antenna array, and $\mathbf{h}_{p}$ is the $p^{\text {th }}$ row of the $(P \times L)$-dimensional channel transfer matrix $\mathbf{H}$. Thus, during the whole Markov processing, the SA MUD algorithm is to choose the specific $L$-symbol vector $\mathbf{x}$, which minimizes the OFM given in (10). The Markov process begins with a random initial vector $\mathbf{x}_{0}$, and its OFM evaluation is $f\left(\mathbf{x}_{0}\right)$, since [10] tells us the SA algorithm doesn't lie on $\mathbf{x}_{0}$, in this paper $\mathbf{x}_{0}=\hat{\mathbf{x}}_{M M S E}$. Metropolis acceptation rule illustrated in dashed frame of Fig.2 is core of the SA algorithm and explained in detail below. Supposing $\mathbf{x}_{n}$ is the $n^{\text {th }}$ iterative trial vector, and the responding evaluation is $f\left(\mathbf{x}_{n}\right)$; a new trial vector $\mathbf{x}_{n+1}$ is generated randomly from $\mathbf{x}_{n}$, and $f\left(\mathbf{x}_{n+1}\right)$ is its evaluation. If $f\left(\mathbf{x}_{n+1}\right)<f\left(\mathbf{x}_{n}\right), \mathbf{x}_{n+1}$ is accepted as the new starting point for the next iteration. Contrarily, if $f\left(\mathbf{x}_{n+1}\right) \geq f\left(\mathbf{x}_{n}\right), \mathbf{x}_{n+1}$ is accepted with the probability given by [9]

$$
\operatorname{Pr}=\exp \left(-\frac{\Delta f}{T_{k}}\right),
$$

where $\Delta f=f\left(\mathbf{x}_{n+1}\right)-f\left(\mathbf{x}_{n}\right)$, and $T_{k}$ is the temperature of the $k^{\text {th }}$ Cooling Iteration. If $r<\operatorname{Pr}$ where $r$ is a pseudo-random number in the interval $[0,1], \mathbf{x}_{n+1}$ is also accepted, however if the contrary occurs, $\mathbf{x}_{n+1}$ is refused. Then the next iteration begin with vector $\mathbf{x}_{n}$ or the new trial vector $\mathbf{x}_{n+1}$, if $\mathbf{x}_{n+1}$ is refused or accepted, respectively. Such iteration is thus called as a Metropolis Iteration.

\section{B. Trial Vector Generation Scheme}

As discussed in Section III-A, at the beginning of the new Metropolis Iteration $n$, a new trial vector $\mathbf{x}_{n+1}$ is generated randomly from $\mathbf{x}_{n}$. [11] claimed that in MMSE-GA MUD, when considering a specific received symbol, the adjacent constellation symbols are more likely to be the transmitted symbol than the more distant ones, and proposed a Biased Q-Function Based Mutation (BQM) scheme. However, when considering it as a combinatorial optimization problem, we can not derive the same conclusion. Not as GA searches the optimum vector along multi-direction based on population, yet the SA algorithm grabbles along a Markov chain based on every single new vector $\mathbf{x}_{n+1}$. That is to say, how to generate the new trial vector is very important, deciding whether the optimum solution can be found in the finite length of Markov chain. Obviously, according to (2), we can see clearly that, the $l$-element $x^{l}(l=1, \ldots, L)$ of the new trial vector $\mathbf{x}_{n+1}$ is the estimated symbol transmitted by the $l^{\text {th }}$ user, belonging to $M_{c}$. When $\mathbf{x}_{n}$ varies to $\mathbf{x}_{n+1}$, two aspects can be considered: which user is elected to change, and which legitimate point of the elected user should be mutated to. To the best of our knowledge, no open literature at the time of writing can cope with this problem well. In this treatise, UM based trial

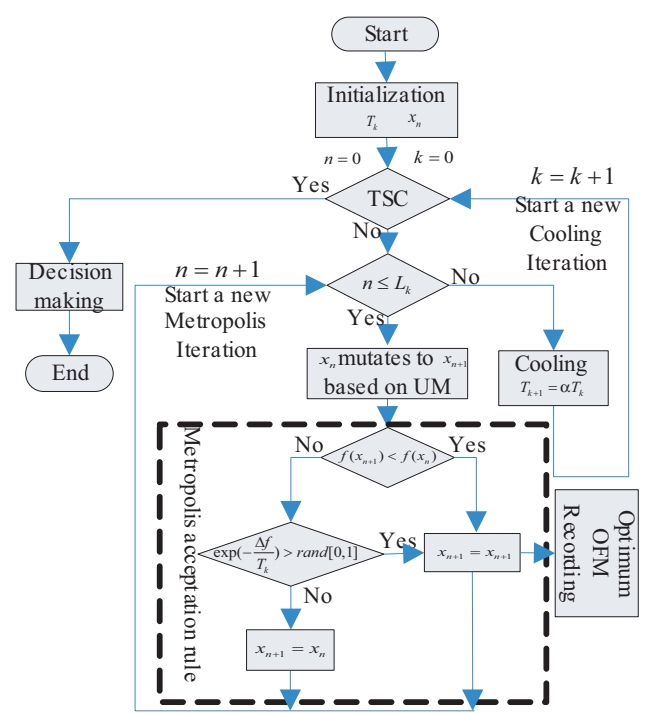

Fig. 2. Flow chart of the SA MUD.

vector generation scheme is brought forward. Specifically, considering without any assistant information, in order to ensure sufficient diversity acquired in the whole Markov chain and to prevent from being trapped at local optima, no one user or legitimate point should be preferred. In another saying, every user has equal chance to be elected to change, and the original point of elected user will mutate to all the other candidate symbols in $M_{c}$ with a same transition probability.

\section{The Optimum OFM Recording}

If the total length of Markov chain is finite, the last returned solution is not always the optimum, so the optimal solution should be recorded in case of being lost during cooling process. Once a new trial vector $\mathbf{x}_{n}$ is generated, comparing its OFM evaluation $f\left(\mathbf{x}_{n}\right)$ with that of the optimum $\mathbf{x}_{\text {optimum }}$, which is initialized as $\hat{\mathbf{x}}_{M M S E}$, if $f\left(\mathbf{x}_{n}\right)<f\left(\mathbf{x}_{\text {optimum }}\right)$, then $\mathbf{x}_{\text {optimum }}=\mathbf{x}_{n}$. If the contrary occurs, $\mathbf{x}_{\text {optimum }}$ will keep.

\section{CS Designing}

1) Initial temperature $T_{0}: T_{0}$ should be determined in such a way that all possible transitions could be made, i.e. $T_{0}$ is a temperature satisfying $\exp \left(-\frac{\Delta f}{T_{0}}\right) \cong 1$ for all $\Delta f$. Specifically, in practical use, a trial set $M_{t}$ containing $\left(\frac{1}{3} \sim \frac{1}{2}\right) \cdot 2^{m L}$ number of vectors is generated firstly, which is composed of $\mathbf{x}_{0}$ and its alterations. In this paper, the size of $M_{t}$ is $\frac{1}{2} \cdot 2^{m L}$ and $\mathbf{x}_{b} \triangleq \arg \min _{\mathbf{x} \in M_{t}} f(\mathbf{x}), \mathbf{x}_{w} \triangleq \arg \max _{\mathbf{x} \in M_{t}} f(\mathbf{x})$. we adopt the method of [12] which suggests the following

$$
T_{0}=\frac{\Delta f_{\max }}{\ln \left(X_{0}\right)},
$$

where $\Delta f_{\max } \triangleq f\left(\mathbf{x}_{b}\right)-f\left(\mathbf{x}_{w}\right)$, and $X_{0}$ is the rate of acceptance, in context of our proposed SA MUD algorithm, $X_{0}=0.5$ is invoked. 
2) Temperature attenuation function: The rate of cooling has a direct effect on the total number of iterations required at each temperature level. A simple rule $T_{k+1}=\alpha T_{k}$ has been widely used in practice [12], and $\alpha$ is a constant. $\alpha=0.85$ as experiential choice is invoked in context of this contribution.

3) The length of Markov chain $N_{k}$ at temperature $T_{k}$ : Not only temperature attenuation function but also the length of Markov chain $N_{k}$ at temperature $T_{k}$ decides the algorithm convergence speed and performance. The strategy of low temperature attenuation rate and relative short $N_{k}$ is adopted in this paper. [10] illuminated that for briefness $N_{k}$ can be a constant $N$ independent of temperature, which is usually expressed as polynomial of optimization problem variables ( $\mathrm{L}$ in this paper), e.g. $N=\gamma \cdot L$, where $\gamma$ is the polynomial coefficient, saying 50, 100, etc. For different values of $\gamma$ the simulation results in Section IV demonstrate the diverse performances of the proposed SA MUD algorithm.

4) Twofold Stopping Criteria (TSC): The stopping criterion is generally set in such a way that at the optimal point the expected improvements in the OFM evaluations become negligible. In the discourse of our proposed SA MUD algorithm, we adopt two stopping criteria [12], which have been used in practice widely. The first one assumes the number of temperature level $T_{f}$ is fixed for the cooling process, i.e. if temperature is attenuated to $T_{f}$, then the process stops; and the second one is based on the rate of improvement in the OFM evaluations, i.e. if the OFM evaluations don't improve for $C_{N}$ consecutive cooling iterations, the algorithm stops. In this paper, $T_{f}=0.05$ and $C_{N}=3$. In the low-user-loaded scenario, the optimal OFM evaluations may be the same in two or three consecutive cooling iterations. In this sense, when the same OFM evaluations are obtained, the cooling process should be stopped, so that the optimum solution can be reached within finite cooling iterations. By contrast, in the high-userloaded scenario, in order to achieve the optimum solution, more cooling iterations are needed, until the temperature is cooled to $T_{f}$, which is the smaller the better.

\section{SIMULATION RESULTS}

In this section, we characterize the performance of the proposed SA MUD in synchronous SDMA system, and the transmission is subject to Narrow Band Uncoded Rayleigh Fading (NBURF) channel. If not explicitly stated, it was assumed that the channel's transfer functions were perfectly known.

\section{A. BQM versus $U M$ in $S A M U D$}

Fig.3 depicts the BER performance achieved by two different trial vector generation schemes, and the performance of ML MUD is also provided for reference benchmark. As seen in Fig.3, UM-aided SA MUD outperforms BQM-aided scheme, which is introduced in [11] but used in GA MUD. As mentioned in Section III-B, UM scheme ensures sufficient diversity acquired in the whole Markov chain and prevents from being trapped at local optima, so the performance is better.

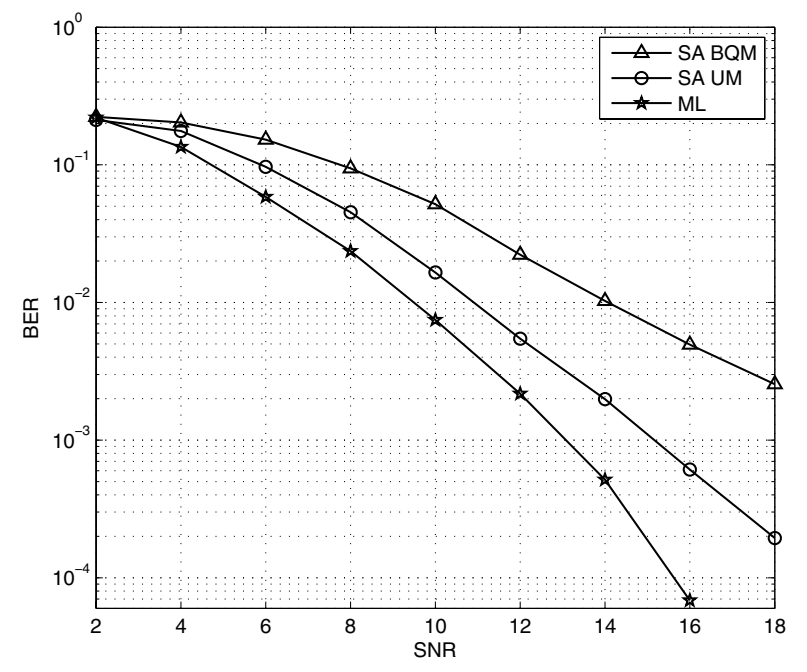

Fig. 3. BER versus SNR performance comparison of SA MUD in SDMA system invoking BQM or UM, while employing a QPSK scheme for transmission on NBURF channel, where $L=8$ users are supported with the aid of $P=6$ receiver antenna elements. $N=100 \cdot L$, and the other parameters are the same as mentioned in the context.

\section{B. Performance versus SNR Comparison between GA MUD and SA MUD}

Iterative GA MUD in TTCM-MMSE-SDMA-OFDM system is advocated in [11], and its simulation results showed that, if the population size $X$ and/or the number of generations $Y$ are sufficiently high, the GA's final solution approaches the optimum. As a contrast, in this contribution GA MUD is also simulated in the same condition as SA MUD, but not as the simulation condition employed in [11]. From the result of Fig.4, MMSE-aided GA MUD yields high residual error. Furthermore, when the number of $X$ and/or $Y$ are increased, the performance of the GA MUD scheme is improved, but still suffer from an error floor, so we can conclude that, the nearML performance of GA MUD in [11] is achieved substantially benefiting from coding and iteration gains. Fortunately, Fig. 4 also shows that, without any extra gains, SA MUD exhibits obvious performance improvement, especially in high SNR scenario. Likewise, Fig.4 reveals the trend of approaching ML MUD performance, as $\gamma$ taking 50 and 100, respectively.

\section{Complexity versus Number of Users Comparison between SA MUD and ML MUD}

In [7] the complexity of GA MUD is portrayed with the number of OFM evaluations according to (10), which brings inspiration to derive the complexity of SA MUD. When the cooling process is stopped according to the twofold stop criteria introduced in Section III-D and $T_{k}$ was not attenuated to $T_{f}$, defining the temperature of the last cooling iteration as $T_{s}$, thus the total number of cooling iterations is formulated as $K=\left[\log _{\alpha}\left(\frac{T_{s}}{T_{0}}\right)\right]$, where $T_{0}$ and $\alpha$ are the initial temperature and temperature attenuation coefficient, respectively. Consequently, SA MUD needs the number of $(K \cdot \gamma \cdot L)$ OFM 


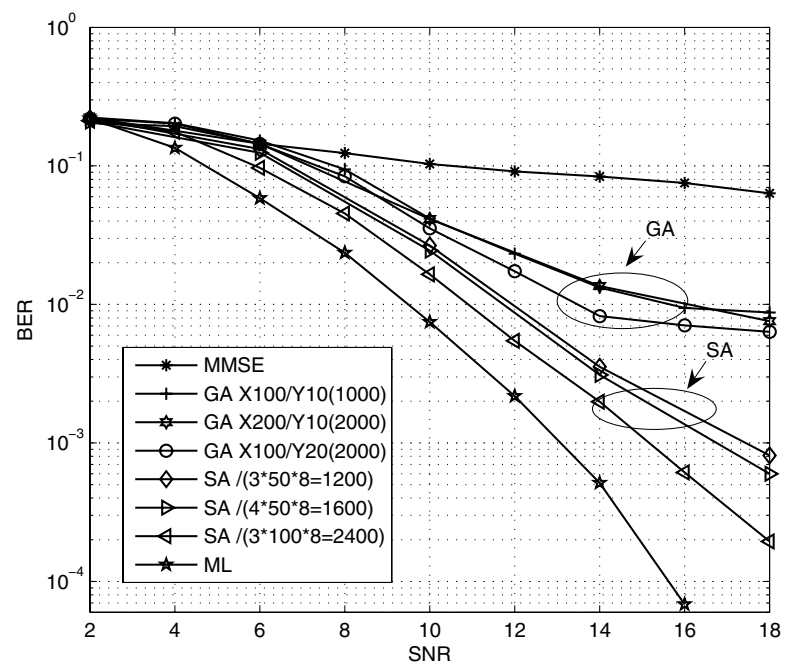

Fig. 4. BER versus SNR performance comparison of SA MUD and GA MUD [11] in SDMA system, while employing a QPSK scheme for transmission on NBURF channel, where $L=8$ users are supported with the aid of $P=6$ receiver antenna elements. The other parameters are the same as mentioned in the context.

evaluations to return the suboptimum solution, and GA MUD requires $(X \cdot Y)$ number OFM calculations, while ML MUD returns the optimum solution at the cost of consuming $2^{m L}$ number OFM evaluations.

In Fig.5, we compare both the ML MUD and the SA MUD in terms of their complexity, i.e. the number of OFM computations. As a specific user load, we always select an appropriate SA-aided scheme for comparison, which suffers from an appropriate $1 \mathrm{~dB}$ SNR loss at the BER of $10^{-3}$ compared to the ML-aided system. As shown in Fig.5, the MLaided system imposes an exponentially increasing complexity on the order of $O\left(2^{m L}\right)$, when the number of users increases, while the complexity of the SA- and GA-aided system required for maintaining a near-optimum performance increases only slowly. Moreover, the complexity of GA MUD is also offered in Fig.5, from which we can see that SA MUD imposes a little lower complexity than GA-aided scheme, and presents better performance.

\section{SUMMARY AND DISCUSSION}

MUD problem in synchronous SDMA system is a typical combinatorial optimization problem, i.e. it is an NP problem. In this paper, a novel SA based MUD scheme is proposed for uncoded SDMA system. As a contrast, GA MUD [11] is also simulated in the same conditions as SA MUD. From the investigations conducted, we conclude that, in both underloaded and overloaded scenarios, SA MUD outperforms GA MUD and imposes lower complexity in synchronous SDMA system without coding and iteration gains, and is capable of achieving a similar performance to that attained by ML MUD.

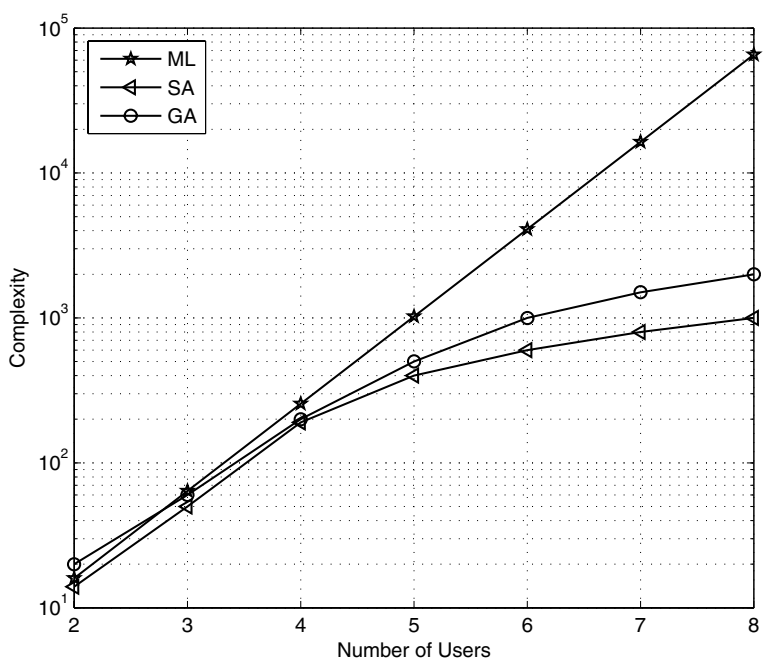

Fig. 5. Comparison of the MUD complexity in terms of the number of OFM evaluations. The number of receiver antenna elements employed is equivalent to the number of users supported, i.e. $\mathrm{L}=\mathrm{P}$.

\section{REFERENCES}

[1] L. Hanzo, M. Munster, B. J. Choi, and T. Keller, OFDM and MC-CDMA for Broadband Multi-User Communications, WLANs and Broadcasting . Piscataway, NJ: IEEE Press/Wiley, 2003.

[2] M. Jiang and L. Hanzo, "Multiuser MIMO-OFDM for Next-Generation Wireless Systems," Proc. IEEE, vol. 95, pp. 1430-1469, 2007.

[3] K. Yen and L. Hanzo, "Genetic-algorithm-assisted multiuser detection in asynchronous CDMA communications" Vehicular Technology, IEEE Transactions on, vol. 53, pp. 1413-1422, 2004.

[4] C. Ergun and K. Hacioglu, "Multiuser detection using a genetic algorithm in CDMA communications systems," Communications, IEEE Transactions on, vol. 48, pp. 1374-1383, 2000.

[5] K. Yen and L. Hanzo, "Hybrid genetic algorithm based detection schemes for synchronous CDMA systems," presented at Vehicular Technology Conference Proceedings, 2000. VTC 2000-Spring Tokyo. 2000 IEEE 51st, 2000.

[6] M. Jiang, J. Akhtman, and L. Hanzo, "Soft-Information Assisted NearOptimum Nonlinear Detection for BLAST-type Space Division Multiplexing OFDM Systems," Wireless Communications, IEEE Transactions on, vol. 6, pp. 1230-1234, 2007.

[7] M. Jiang and L. Hanzo, "Improved hybrid MMSE detection for turbotrellis-coded modulation-assisted multi-user OFDM systems," Electronics Letters, vol. 40, pp. 1002-1003, 2004.

[8] M. Jiang, T. Lestable, and Y. Cho, "Uplink Multi-User MIMO OFDM Enhancement Using Genetically Improved Turbo Receiver," presented at Vehicular Technology Conference, 2007. VTC-2007 Fall. 2007 IEEE 66th, 2007.

[9] Y. Wang, W. Yan, and G. Zhang, "Adaptive simulated annealing for the optimal design of electromagnetic devices," Magnetics, IEEE Transactions on, vol. 32, pp. 1214-1217, 1996.

[10] Y. Xin and L. Guojie, "General simulated annealing," Journal of Computer Science \& Technology, vol. 6, pp. 329-338, 1991.

[11] J. Ming, N. Sooh Xin, and L. Hanzo,"Hybrid iterative multiuser detection for channel coded space division multiple access OFDM systems" Vehicular Technology, IEEE Transactions on, vol. 55, pp. 115-127, 2006.

[12] R. Romero, R. A. Gallego, and A. Monticelli, "Transmission system expansion planning by simulated annealing," Power Systems, IEEE Transactions on, vol. 11, pp. 364-369, 1996. 